\title{
Placenta growth factor and neuropilin-1 collaborate in promoting melanoma aggressiveness
}

\author{
ELENA PAGANI $^{{ }^{*}}$, FEDERICA RUFFINI ${ }^{1 *}$, GIAN CARLO ANTONINI CAPPELLINI ${ }^{2}$, \\ ALESSANDRO SCOPPOLA $^{2}$, CRISTINA FORTES ${ }^{3}$, PAOLO MARCHETTI $^{4}$, \\ GRAZIA GRAZIANI $^{5}$, STEFANIA D'ATRI ${ }^{1}$ and PEDRO MIGUEL LACAL ${ }^{1}$
}

\author{
${ }^{1}$ Laboratory of Molecular Oncology, ${ }^{2}$ Department of Oncology and Dermatological Oncology, and ${ }^{3}$ Epidemiology Unit, \\ 'Istituto Dermopatico dell'Immacolata'- IRCCS; ${ }^{4}$ Department of Oncology, Sant'Andrea Hospital, University of Rome \\ 'La Sapienza'; ${ }^{5}$ Department of Systems Medicine, University of Rome 'Tor Vergata', Rome, Italy
}

Received September 11, 2015; Accepted October 20, 2015

DOI: 10.3892/ijo.2016.3362

\begin{abstract}
The placenta growth factor (PIGF) is a member of the vascular endothelial growth factor (VEGF) family, which shares with VEGF-A the tyrosine kinase receptor VEGFR-1 and the co-receptor neuropilin-1 (NRP-1). In melanoma models, PIGF enhances tumour growth and neovessel formation, whereas NRP-1 promotes the metastatic process. Increased secretion of PIGF and expression of NRP-1 have also been involved in intrinsic or acquired resistance to anti-angiogenic therapies. In this study we investigated whether PlGF and NRP-1 cooperate in promoting melanoma aggressiveness independently of VEGFR-1. For this purpose, the melanoma cell clones M14-N, expressing NRP-1 and lacking VEGFR-1, and M14-C, devoid of both receptors, were used. M14-N cells are characterized by an invasive phenotype and vasculogenic mimicry, whereas M14-C cells possess a negligible invasive capacity. The results indicated that M14-N cells secrete higher levels of PIGF than M14-C cells and that PIGF is involved in the invasion of the extracellular matrix (ECM) and vasculogenic mimicry of M14-N cells. In fact, neutralizing antibodies against PIGF reverted ECM invasion in response to PIGF and markedly reduced the formation of tubule-like structures. Moreover, M14-N cells migrated in response to PIGF and chemotaxis was specifically abrogated by antiNRP-1 antibodies, demonstrating that PlGF directly activates NRP-1 in the absence of VEGFR-1. We also compared the levels of PIGF in the plasma of patients affected by metastatic melanoma with those of healthy donors and evaluated whether
\end{abstract}

Correspondence to: Dr Pedro Miguel Lacal, Laboratory of Molecular Oncology, 'Istituto Dermopatico dell'Immacolata'IRCCS, Via dei Castelli Romani 83/85, I-00040 Rome, Italy

E-mail: p.lacal@idi.it

${ }^{*}$ Contributed equally

Key words: PlGF, neuropilin-1, melanoma, bevacizumab, vasculogenic mimicry
PIGF levels could be affected by a bevacizumab-containing chemotherapy regimen. Melanoma patients showed a 20-fold increase in plasma PIGF and the bevacizumab-containing regimen induced a reduction of VEGF-A and in a further increase of PlGF. In conclusion, our studies suggest that the activation of NRP-1 by PlGF directly contributes to melanoma aggressiveness and represents a potential compensatory proangiogenic mechanism that may contribute to the resistance to therapies targeting VEGF-A.

\section{Introduction}

Melanoma is a highly aggressive neoplasm, characterized by early metastatic spreading and poor response to chemotherapy and radiotherapy. Despite the recent advances in the treatment of the metastatic disease with the approval of monoclonal antibodies that block immune checkpoints (ipilimumab, nivolumab and pembrolizumab) and inhibitors of BRAF (vemurafenib and dabrafenib) or MEK (trametinib), the prognosis of the advanced disease remains poor. Melanoma progression is favoured by the formation of new vessels from the pre-existing ones (angiogenesis), and by the ability of tumour cells to invade the extracellular matrix (ECM) and to form structures similar to blood vessels (vasculogenic mimicry).

A key role in the angiogenic process is played by members of the vascular endothelial growth factor (VEGF) family, and in particular by the VEGF-A and the placenta growth factor (PlGF) (1,2), which can be released by the tumour cell itself. VEGF-A interacts with two membrane tyrosine kinase receptors (3): VEGFR-1 (involved in the activation of cell migration and in the modulation of the signalling transduced by the VEGFR-2) and VEGFR-2 (mainly responsible for the signal transduction that mediates the effects of VEGF-A). Conversely, PlGF binds only to VEGFR-1, promoting migration, proliferation and survival of endothelial cells.

The expression of PIGF is upregulated in several types of human cancers and is associated with a poor prognosis $(4,5)$. PIGF is capable of transducing its own signals through the phosphorylation of tyrosine residues within VEGFR-1, which are distinct from those phosphorylated upon stimulation of the receptor by VEGF-A (6). PIGF expression in the skin of 
transgenic mice inoculated with syngeneic melanoma cells, favours tumour growth and mobilization of endothelial and hematopoietic stem cells. In the same murine model PIGF also increases the number and size of melanoma-associated vessels and the formation of pulmonary metastases (7). Moreover, PIGF promotes tumour cell invasion of the ECM and enhances the activity of selected matrix metalloproteinases (7). Interestingly, PIGF plays also a role in melanoma cell resistance to chemotherapy through a pathway that involves $\mathrm{NF}-\kappa \mathrm{B}$ activation (8).

The VEGFR-1 is expressed in endothelial cells during vessel formation and remodelling, in macrophages and in myoepithelial cells, favouring cell migration and survival (9-11). Moreover, it is frequently expressed in a variety of human tumours where it predicts poor prognosis and recurrence $(1,9)$. In cancer cells, VEGFR-1 signalling has been shown to inhibit apoptosis and to induce chemoresistance $(1,12,13)$. Indeed, VEGFR-1 neutralization has been reported to prolong survival of tumour-bearing mice (14). We previously demonstrated that PIGF and VEGFR-1 are co-expressed in a large number of human melanoma cell lines (15) and, together with other groups, suggested that the interaction of PIGF with VEGFR-1 might modulate cellular pathways important for melanoma cell proliferation, apoptosis and invasiveness (7,15-18).

Another receptor, capable of binding VEGF-A and PIGF is neuropilin-1 (NRP-1), a membrane protein devoid of tyrosine kinase activity. Through the interaction with VEGF-A, NRP-1 acts as a co-receptor, enhancing the signal transmitted by VEGFR-1 and VEGFR-2 (2,4,19-21). Moreover, although the intracellular domain of NRP-1 is not able to directly transmit a signal, its interaction with intracellular regulators allows NRP-1 to activate signal transduction pathways independently of tyrosine kinase VEGF-A receptors (22). In this regard, PIGF has been recently shown to activate MAPK and AKT signalling and promote tumour cell survival by interacting with NRP-1 in the absence of VEGFR-1 (23). Actually, a wide variety of human malignancies (cell lines and tissue samples) express NRP-1 and experimental evidence indicates an association between the expression of NRP-1 and tumour growth, invasiveness, angiogenesis and poor prognosis (24).

Given the key role played by VEGFs and VEGFRs in the process of angiogenesis, intensive preclinical and clinical investigation has been focused on the development of targeted drugs that interfere with their activation or signal transduction (25). To date, two angiogenic monoclonal antibodies have been approved for cancer treatment: bevacizumab, a humanized antibody that targets VEGF-A, preventing its interaction with both VEGFR-2 and VEGFR-1, and ramucirumab, a fully human antibody against VEGFR-2. Several small molecules that interact with the catalytic domain of VEGFRs inhibiting their tyrosine kinase activity, are also available. With few exceptions, these agents are mostly used in combination with chemotherapy for the treatment of a variety of solid tumours (26).

Unfortunately, the benefits obtained from anti-angiogenic therapies have short duration, due to acquired resistance. The mechanisms responsible for resistance to anti-VEGF-A therapies $(26,27)$ include, among others, the formation of blood vessels by mechanisms alternative to angiogenesis (intussusception, co-option, vasculogenic mimicry), the production of angiogenic factors different from VEGF-A (e.g., PlGF) and the high expression of VEGFR-1 (in tumour or endothelial cells and macrophages) or NRP-1 (in tumour cells). Interestingly, although melanoma is a highly vascularised tumour and potentially amenable to treatment with anti-angiogenic agents, none of the anti-VEGF-A or VEGFR inhibitors have been approved so far for the therapy of this malignancy.

In this study we investigated whether PlGF and NRP-1 cooperate in promoting melanoma aggressiveness and in the acquisition of resistance to anti-VEGF-A therapies. Our results indicate that in melanoma cells lacking VEGFR-1, PIGF is able to stimulate ECM invasion and vasculogenic mimicry through the sole activation of NRP-1. We also found that plasma levels of PlGF are higher in patients with metastatic melanoma than in healthy subjects and further increase during treatment with a bevacizumab-containing chemotherapy regimen.

\section{Materials and methods}

Melanoma cell lines and culture conditions. The origin and culture conditions of the human umbilical vein endothelial cells (HUVECs), used as control, and of the human melanoma cell lines utilised in the study (GR-Mel, derived from a primary tumour, M14 and WM266-4, both derived from metastatic lesions), as well as their characterization regarding the expression of VEGFR-1 and NRP-1, have been described elsewhere (15). The cell clones M14-N and M14-C were derived from the M14 cell line, as previously described $(28,29)$. The M14-N clone expresses NRP-1 and secretes high levels of VEGF-A and MMP-2, but lacks VEGFR-2 expression (28). The control M14-C clone is devoid of the expression of VEGFR-2 and NRP-1 and secretes lower amounts of VEGF-A and MMP-2 $(28,29)$. Culture media and reagents were purchased from Sigma-Aldrich (St. Louis, MO, USA) and fatty acid-free bovine serum albumin (BSA) was from Roche Diagnostics GmbH (Mannheim, Germany). The recombinant human PIGF used in the functional assays was from R\&D Systems, Inc. (Minneapolis, MN, USA).

Western blot analysis. VEGFR-1 and NRP-1 expression in melanoma cells was evaluated in cell extracts by western blot analysis as described (28). The anti-human NRP-1 mouse monoclonal antibody (A-12), the rabbit polyclonal antibodies anti-human VEGFR-1 (C17) and anti-human $\beta$-tubulin (H-235, used as a loading control) were purchased from Santa Cruz Biotechnology, Inc. (Santa Cruz, CA, USA) and utilized at the final concentration of $0.2 \mu \mathrm{g} / \mathrm{ml}$.

Migration and invasion assays. In vitro migration assays were performed using Boyden chambers equipped with $8-\mu \mathrm{m}$ pore diameter polycarbonate filters (Nuclepore; Whatman Inc., Clifton, NJ, USA), coated with $5 \mu \mathrm{g} / \mathrm{ml}$ gelatin (30). Briefly, melanoma cells were collected from continuous cultures, washed, suspended in migration medium $(1 \mu \mathrm{g} / \mathrm{ml} \mathrm{heparin} / 0.1 \%$

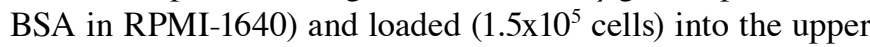
compartment of the Boyden chambers. Migration medium, with or without $50 \mathrm{ng} / \mathrm{ml} \mathrm{PlGF}$, was added to the lower compartment and the chambers were incubated at $37^{\circ} \mathrm{C}$ in a $\mathrm{CO}_{2}$ atmosphere for $18 \mathrm{~h}$. The filters were then removed from 
the chambers and cells were fixed in ethanol and stained in crystal violet. The migrated cells, attached to the lower surface of the filters, were counted under the microscope. Twelve high-magnification microscopic fields (magnification, x100), randomly selected on triplicate filters, were scored for each experimental condition.

In vitro ECM invasion by melanoma cells was analysed in Boyden chambers as described for the migration assay, except that polycarbonate filters were coated with $20 \mu \mathrm{g}$ of the commercial basement membrane matrix Matrigel (Trevigen, Gaithersburg, MD, USA) (18) and $1 \times 10^{5}$ cells/chamber were allowed to invade for $2 \mathrm{~h}$.

In selected experiments, migration or invasion assays were performed in the presence of antibodies against PIGF (mouse monoclonal anti-human antibody, MAB264 from R\&D Systems, Inc.), NRP-1 (rabbit polyclonal anti-human antibody, H286 from Santa Cruz Biotechnology, Inc.), and VEGFR-1 (goat polyclonal anti-human antibody; AF321) and VEGFR-2 (goat polyclonal anti-human antibody; AF357) (both from R\&D Systems, Inc.) or the corresponding control immunoglobulins (mouse IgG1 isotype control from R\&D Systems, Inc.). Melanoma cells were pre-incubated with the antibody under investigation for $30 \mathrm{~min}$ at room temperature in a rotating wheel and, then, loaded in the Boyden chambers without removing the antibody.

ELISA quantification of VEGF and PlGF levels. For the evaluation of PIGF secretion by melanoma cell lines, semi-confluent cell cultures were incubated for $24 \mathrm{~h}$ in $0.1 \% \mathrm{BSA} / \mathrm{RPMI}-1640$ medium without serum. Thereafter, culture supernatants were collected and concentrated at least 10-fold in Centriplus concentrators (Amicon, Beverly, MA, USA). Cells were detached from the flasks with a PBS/EDTA solution and the total cell number/culture was recorded to normalise cytokine secretion.

For the evaluation of PIGF and VEGF-A levels in the plasma of melanoma patients and healthy donors (see below), plasma was separated from peripheral blood and cryopreserved at $-80^{\circ} \mathrm{C}$.

The amounts of VEGF or PIGF present in the culture medium or in plasma samples were determined using commercial ELISA kits (R\&D Systems, Inc.), following the instructions from the manufacturer.

Differentiation of melanoma cells in tubule-like structures. The analysis of tubule-like structures on Matrigel was performed as previously described (29). Melanoma cells were suspended in complete culture medium $\left(1 \times 10^{5}\right.$ cells in $\left.0.5 \mathrm{ml}\right)$, dispensed onto $100 \mu \mathrm{l}$ of solidified Matrigel (diluted 1:3 in serum-free RPMI-1640 medium) and incubated for $6 \mathrm{~h}$ at $37^{\circ} \mathrm{C}$ in a $5 \% \mathrm{CO}_{2}$ environment. Afterwards, the plates were photographed using a Leica inverted microscope equipped with a Canon digital camera (PowerShot G5). The formation of tubule-like structures was quantified by counting the number of cell intersections in ten different microscopic fields for each experimental group (x50 magnification).

Studies in patients with metastatic melanoma. Plasma levels of VEGF-A and PIGF were measured in eight healthy donors and eight melanoma patients enrolled in a phase II study evaluating bevacizumab (10 $\mathrm{mg} / \mathrm{kg}$ days 1 and $15, \mathrm{q} 28)$ in combination with paclitaxel $\left(60 \mathrm{mg} / \mathrm{m}^{2}\right.$, days 1,8 and $\left.15, \mathrm{q} 28\right)$ and carboplatin (AUC 2, days 1,8 and 15, q28), as a second-line treatment for metastatic melanoma. The study protocol was approved by the Ethics Committee of the 'Istituto Dermopatico dell'Immacolata'- IRCCS, at the 02/12/08 meeting (Protocol number \#133/EC/2008, Reference number: Register of the Ethics Committee 247/1; Promoter: IDI-IRCCS, Oncology Division, Professor Marchetti; EudraCT code: 2008-06191-30; Protocol: IDI-ONC-3-20080901). Written informed consent was obtained from all the subjects (patients and controls).

The inclusion criteria were as follows: age between 18 and 75 years; histologically confirmed metastatic melanoma progressing after one line of chemotherapy not containing platinum analogues; measurable metastatic disease according to the Response Evaluation Criteria in Solid Tumours (RECIST); ECOG PS $\leq 2$; life expectancy of more than 3 months; adequate blood parameters (leukocytes $>4,000 / \mathrm{mm}^{3}$, neutrophils $>2,000 / \mathrm{mm}^{3}$, platelets $>100,000 / \mathrm{mm}^{3}$, haemoglobin $>10 \mathrm{~g} / \mathrm{dl}$, serum creatinine $<2.5 \mathrm{mg} / \mathrm{dl}$, total bilirubin/GOT/GPT $<1.5 \mathrm{x}$ the upper limit).

The exclusion criteria were as follows: presence of brain metastases, a history of other cancers diagnosed within 5 years (except basal cell skin cancer or carcinoma in situ of the cervix); previous treatment with platinum analogues and/or bevacizumab; concomitant immunotherapy; radiation therapy performed within 28 days before treatment; a history of myocardial infarction, congestive heart failure or heart disease and/or uncontrolled, active infections; acute and/or chronic HIV, hepatitis and/or tuberculosis infections; peripheral neuropathy (higher than grade 2, according to the NCIC-CTC criteria); contraindication to the use of corticosteroids (unstable diabetes mellitus, active stomach ulcer); baseline LDH level $>1.5 \mathrm{x}$ the upper limit of the normal range; pregnancy or breast feeding.

Blood samples were collected during the first cycle of therapy on day 1 before bevacizumab and chemotherapy administration, on day 8 before chemotherapy administration and on day 15 before bevacizumab and chemotherapy administration.

\section{Results}

NRP-1 enhances PlGF-mediated ECM invasion in melanoma cells expressing VEGFR-1. We have previously demonstrated in a murine model that PlGF promotes the invasiveness of melanoma cells through the activation of its tyrosine kinase receptor VEGFR-1 (7). In order to investigate the requirement of NRP-1 for this PlGF function, we initially evaluated the ability of PlGF to stimulate ECM invasion using melanoma cell lines that we had previously characterized for VEGFR-1 and NRP-1 expression by RT-PCR analysis (15) (Fig. 1). M14 cells, lacking VEGFR-1 and expressing very low levels of NRP-1, and GL-Mel cells, expressing VEGFR-1 but lacking NRP-1, did not respond to PIGF. Conversely, WM266-4 cells, expressing both VEGFR-1 and NRP-1, efficiently migrated through the ECM when exposed to the growth factor. In these cells a complete abrogation of melanoma cell invasiveness was observed when the assay was carried out in the presence of a blocking antibody against VEGFR-1, whereas a blocking 


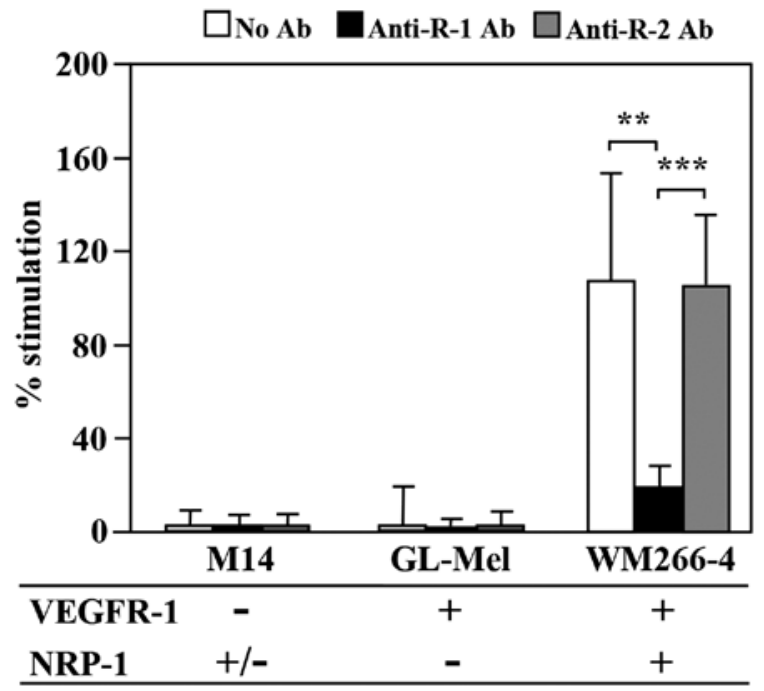

Figure 1. PlGF promotes ECM invasion by melanoma cells. The ability of different melanoma cell lines to invade the ECM in response to PIGF $(50 \mathrm{ng} / \mathrm{ml})$ was tested in the absence or in the presence of $5 \mu \mathrm{g} / \mathrm{ml}$ VEGFR-1 blocking antibodies (anti-R1 Ab) or, as a control, with VEGFR-2 blocking antibodies (anti-R2 Ab). PlGF-induced invasion of ECM was evaluated using Boyden chambers equipped with Matrigel-coated filters. The expression by RT-PCR (+, high; +/-, low; -, no expression) of VEGFR-1 and NRP-1 in each cell line derives from our previously published results (15). Data are expressed as percentage of stimulation of ECM invasion calculated with respect to control cells exposed to culture medium without PIGF. Each value represents the mean \pm SD of at least three independent experiments. ${ }^{* *} \mathrm{P}<0.01$ and ${ }^{* * * *} \mathrm{P}<0.001$, according to Student's t-test analysis.

antibody against VEGFR-2, used as a control, did not have any effect on PlGF-induced invasion (Fig. 1). These results suggested that PlGF strongly induced ECM invasion only when melanoma cells expressed high levels of NRP-1, besides VEGFR-1.

In VEGFR-1 negative melanoma cells PlGF promotes ECM invasion and vasculogenic mimicry through the interaction with NRP-1. We then investigated whether NRP-1 acts only as co-receptor for VEGFR-1 or is also able to initiate by itself a signal transduction after PIGF binding in the absence of VEGFR-1. To this end, we used two previously described cell clones derived from the M14 melanoma cell line: the M14-N cells, characterized by an aggressive phenotype, and the M14-C cells with a negligible invasive capability $(28,31)$. The M14-N cells lack VEGFR-1 and express high levels of NRP-1 protein, whereas the M14-C cells do not express either VEGFR-1 or NRP-1 (Fig. 2A). Evaluation of PIGF secretion in the culture supernatants indicated an increased secretion of this cytokine by M14-N cells as compared to M14-C cells (Fig. 2B). Moreover, PlGF specifically induced ECM invasion by M14-N cells, since an anti-PIGF antibody completely reversed this effect, whereas an unrelated control antibody did not affect the ECM invasion triggered by this cytokine (Fig. 2C and D).

The invasiveness of M14-N cells is strongly hampered by NRP-1 neutralization (28), making it difficult to demonstrate a direct activation of NRP-1 by PlGF. Therefore, the ability of PIGF to activate NRP-1 in M14-N cells was tested using a chemotaxis assay, in which the background migration was very low and the stimulation by this cytokine strongly induced migration (Fig. 3A and B). It should be noted that due to the highly invasive phenotype of M14-N cells, for the invasion assay a short incubation time was sufficient $(2 \mathrm{~h})$, whereas the migration assay required a longer incubation (18 h). Blocking antibodies to PIGF or to the NRP-1 were equally able to completely abrogate PIGF-induced chemotaxis.

A characteristic of highly aggressive cancers, and in particular of melanoma (32), is the ability to form tubule-like structures (vasculogenic mimicry), which provide neoplastic cells with nutrients and facilitate their haematogenous spreading. M14-N cells are able to form tubular structures and this property is strongly dependent on NRP-1 activity (29). Herein we demonstrate that PlGF is directly involved in the formation of tubule-like structures (Fig. 3C and D). In fact, an anti-PIGF blocking antibody markedly reduced cell-to-cell intersections, which were instead unaffected by the treatment with a control antibody (Fig. 3C and D). Thus, the effects of PIGF on vasculogenic mimicry of M14-N cells likely require the activation of NRP-1.

All these data strongly suggested the presence of an autocrine PIGF/NRP-1 loop in highly aggressive melanoma cells.

Analysis of plasma levels of PlGF and VEGF-A in patients with metastatic melanoma. We next investigated whether high PIGF levels could be present in the plasma of patients with metastatic melanoma, and whether the level of this cytokine could be affected by a chemotherapy regimen including bevacizumab to target angiogenesis. To this end, we took advantage from a phase II study carried out to evaluate bevacizumab in combination with carboplatin and paclitaxel as a second-line therapy in patients with metastatic melanoma.

From March 2009 to May 2011, 51 patients with metastatic melanoma progressing after a first line of chemotherapy were examined for eligibility. On the basis of the exclusion criteria, 42 patients ( 21 with secondary brain injury; 10 with ECOG PS $\geq 3$; 8 with no measurable target lesions; 2 with previous heart attack and 1 with previous ictus cerebri) were not enrolled in the study. In addition, one patient withdrew informed consent at the time of the first drug administration. Therefore, only eight patients, five males and three females with a median age of 56 years (38-66), were available for the analysis of VEGF-A and PIGF levels in the blood.

Plasma levels of VEGF-A and PIGF in the eight melanoma patients were determined on day 1,8 and 15 of the first cycle of therapy. Blood samples were collected just before the administration of the drugs. VEGF-A and PIGF levels detected in the plasma collected from patients on day 1 (i.e., baseline values) were compared with those of age- and gender-matched healthy donors. The results showed significant higher amounts of VEGF-A and PIGF in the patients as compared to the healthy controls. Remarkably, we observed a 20 -fold difference in the levels of PIGF between melanoma patients and healthy volunteers (Fig. 4).

For each of the eight patients, VEGF-A and PIGF plasma levels detected on day 8 and 15 were then compared with baseline values (day 1). The results indicated that after a single administration of bevacizumab plus chemotherapy, VEGF-A markedly decreased (day 8) and started to increase again on day 15 , even though its levels remained significantly below the values detected before starting the treatment cycle (Fig. 5). On 
A

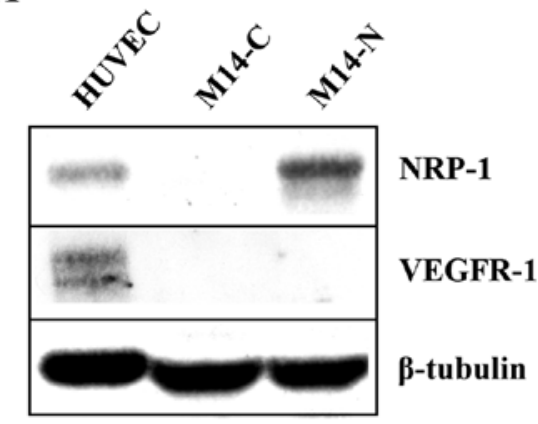

C

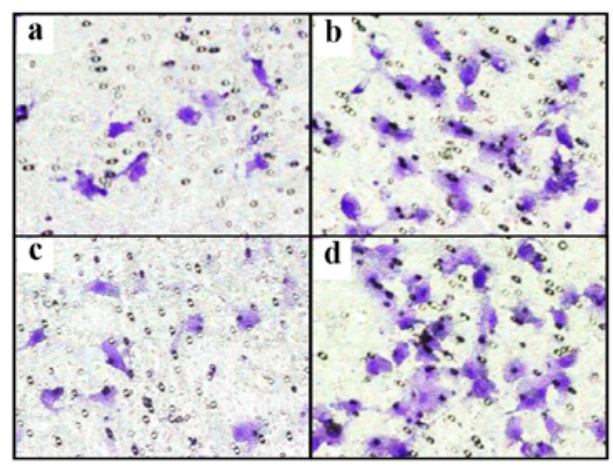

B

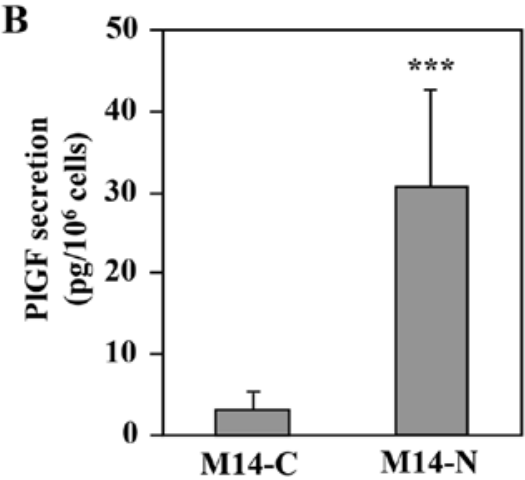

D

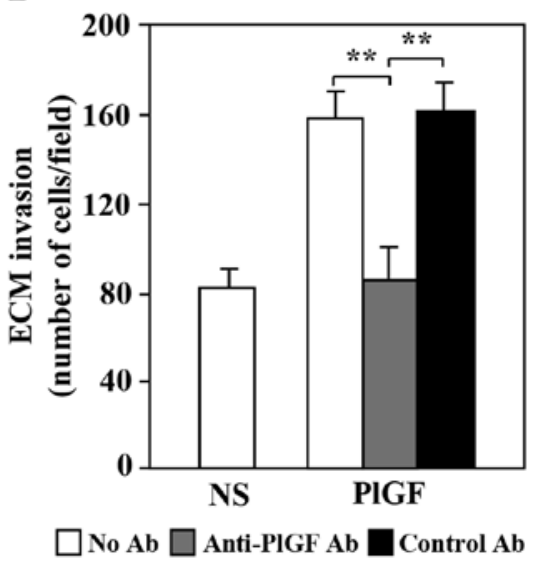

Figure 2. PlGF induces ECM invasion of melanoma cells expressing NRP-1 but lacking VEGFR-1. (A) The expression of NRP-1 and VEGFR-1 proteins in two clones from the human melanoma cell line M14 (M14-C and M14-N clones) was evaluated by western blot analysis utilising antibodies against NRP-1, VEGFR-1 and $\beta$-tubulin (loading control), as indicated in the Materials and methods section. Extracts from HUVEC were used as positive control. (B) PlGF secretion into the culture supernatant of M14 clones was quantified by ELISA. Each value represents the mean \pm SD of eight independent determinations. ${ }^{* * * *} \mathrm{P}<0.001$, according to Student's t-test analysis comparing the amount of PlGF secreted by M14-N cells to that secreted by M14-C cells. (C) P1GF-induced invasion of ECM by M14-N cells was evaluated using Boyden chambers equipped with Matrigel-coated filters. Selected representative microscopic fields (x200 magnification) are shown that correspond to: (a) non-stimulated cells; (b) cells stimulated with $50 \mathrm{ng} / \mathrm{ml} \mathrm{PlGF}$ in the absence of antibodies (Ab); (c) cells stimulated with PlGF in the presence of $5 \mu \mathrm{g} / \mathrm{ml}$ mouse monoclonal anti-PlGF Ab; and (d) cells stimulated with PlGF in the presence of $5 \mu \mathrm{g} / \mathrm{ml}$ control Ab. (D) Quantification of ECM invasion assays described in panel C. Data are expressed in terms of number of invading cells per microscopic field. The results correspond to a representative experiment out of three independent assays. Each value represents the mean \pm SD of 12 fields in triplicate chambers for each experimental condition. ${ }^{* *} \mathrm{P}<0.01$, according to Student's t-test analysis.

the other hand, plasma PIGF significantly increased on day 8 (2.3-fold increase) and remained high until the end of the cycle (3-fold increase) (day 15; Fig. 5).

Overall, the results indicated that patients with metastatic melanoma show measurable levels of PIGF in the plasma and that treatment with bevacizumab induces a further increase of this cytokine.

\section{Discussion}

In the present study we demonstrate for the first time that PIGF promotes ECM invasion and vasculogenic mimicry of human melanoma cells through the activation of NRP-1 and independently of VEGFR-1. These findings strongly suggest a role for PIGF and NRP-1 in melanoma aggressiveness and in the activation of compensatory pro-angiogenic mechanisms that may contribute to innate or acquired resistance to anti-angiogenic therapies targeting VEGF-A.

The involvement of PlGF in melanoma progression has been previously suggested $(7,15-18,33)$ and confirmed by studies performed in a murine transgenic model where high PIGF expression levels were selectively induced in the skin (7). On the other hand, NRP-1, initially considered only a co-receptor of tyrosine kinase receptors for different ligands, has been more recently shown to play an important role in the activation of specific signal transduction pathways independently of other membrane receptors (23,34-36). Our previous data indicate that NRP-1, upon interaction with VEGF-A, may contribute to the melanoma metastatic potential even in the absence of VEGFR-2, stimulating tumour invasiveness and vasculogenic mimicry, which facilitates the haematogenous spreading of cancer cells $(28,29)$. These effects require the triggering of signal transduction pathways involving AKT (28), specific gene expression (31), integrin activation (29) and the secretion of metalloproteinases that degrade the ECM, such as metalloproteinases 2 and $9(28,29,37)$.

Even though disease progression is highly dependent on angiogenesis, melanoma may undergo phenotypic changes which limit the efficacy of anti-VEGF-A agents. Here we demonstrate that in melanoma cells NRP-1 may be activated by PIGF also in the absence of VEGFR-1. Both PIGF and NRP-1 are, therefore, involved in the induction of mechanisms that may result in tumour progression and resistance to anti-angiogenic therapies. 
A

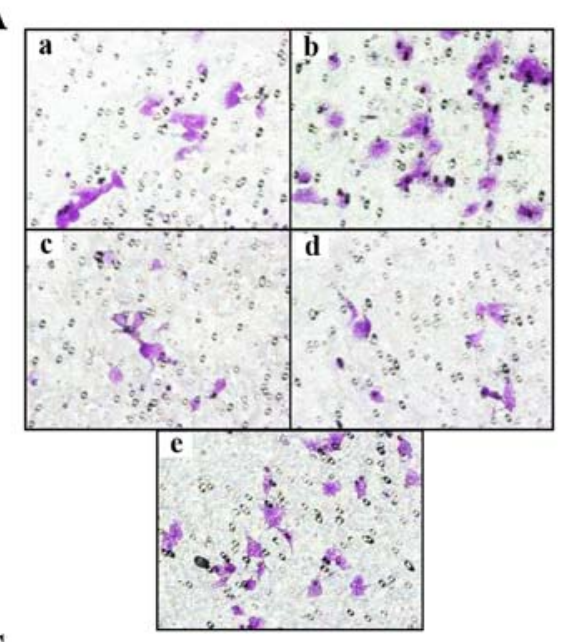

C

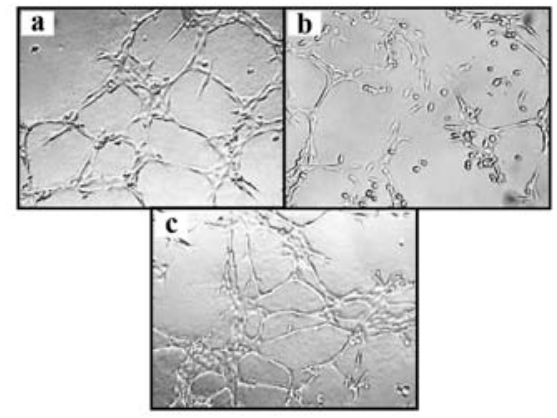

B

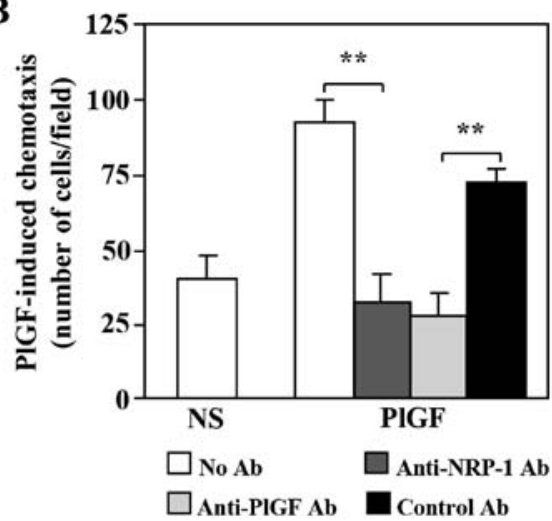

D

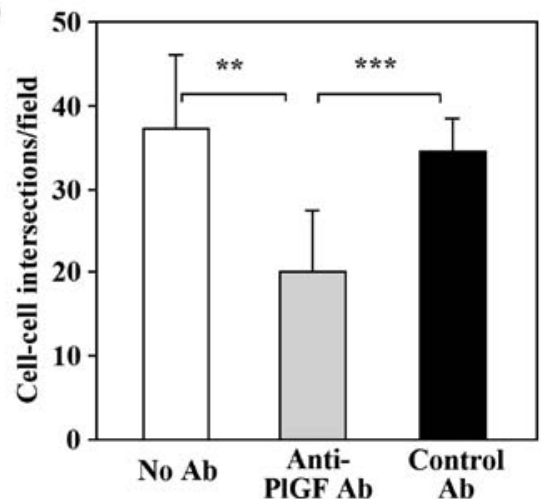

Figure 3. PlGF induces a chemotactic response in M14-N cells through the activation of NRP-1 and is involved in vasculogenic mimicry. (A) The chemotactic response of M14-N cells to PlGF was evaluated using Boyden chambers equipped with gelatine-coated filters. Representative microscopic fields (x200 magnification) are shown that correspond to: non-stimulated cells (a); cells stimulated with $50 \mathrm{ng} / \mathrm{ml} \mathrm{PlGF}$ in the absence of antibodies (Ab) (b); cells stimulated with PlGF in the presence of $5 \mu \mathrm{g} / \mathrm{ml}$ mouse monoclonal anti-PlGF Ab (c); cells stimulated with PlGF in the presence of $5 \mu \mathrm{g} / \mathrm{ml} \mathrm{rabbit} \mathrm{polyclonal} \mathrm{anti-NRP-1}$ $\mathrm{Ab}(\mathrm{d})$; and cells stimulated with PlGF in the presence of $5 \mu \mathrm{g} / \mathrm{ml}$ control $\mathrm{Ab}$ (e). (B) The results of a representative experiment out of three are shown. Data represent the mean number of cells per microscopic field \pm SD of 12 fields in triplicate chambers for each experimental condition. (C) Effect of the mouse monoclonal Ab against PlGF on the ability of M14-N cells to form tubule-like structures on Matrigel. Cells were untreated (a) or pre-incubated with $5 \mu \mathrm{g} / \mathrm{ml}$ of anti-PlGF $\mathrm{Ab}(\mathrm{b})$ or control $\mathrm{Ab}$ (c) for $30 \mathrm{~min}$ in a rotating wheel at room temperature, before seeding them on Matrigel. Tubule-like structure formation was analyzed after $6 \mathrm{~h}$. Photographs from a representative experiment out of three are shown (x50 magnification). (D) The number of cell intersections formed by the tubule-like structures obtained in the experiment shown in panel $\mathrm{C}$ was counted in ten different microscopic fields for each experimental group. Data represent the mean $\pm \mathrm{SD}$ of a representative experiment. ${ }^{* *} \mathrm{P}<0.01$ and ${ }^{* * * *} \mathrm{P}<0.001$, according to Student's $\mathrm{t}$-test analysis.
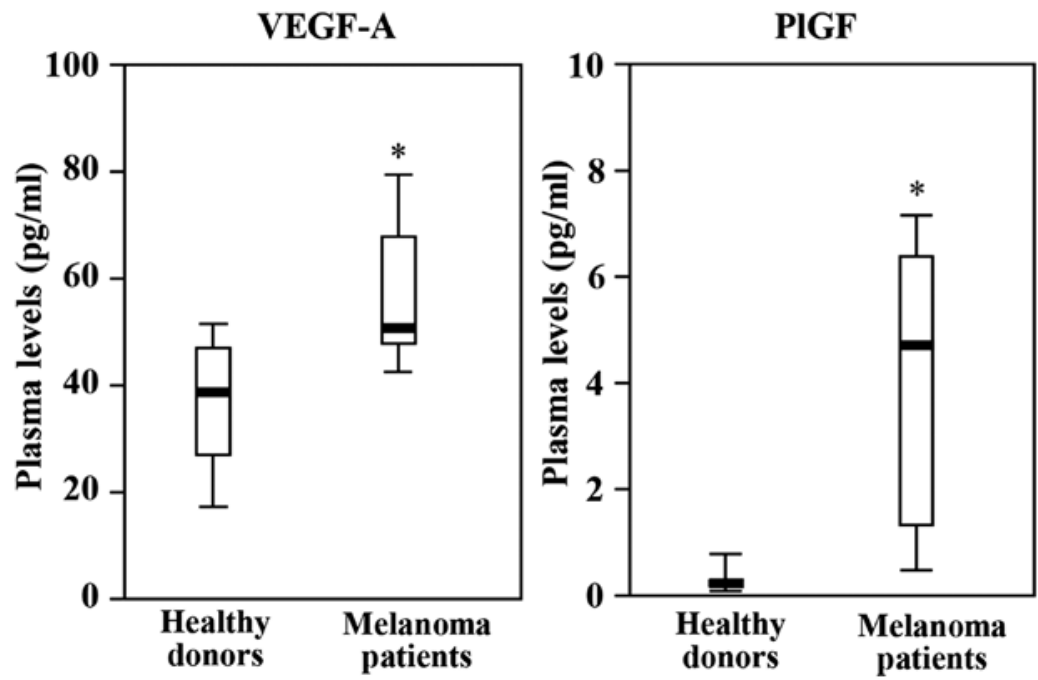

Figure 4. Evaluation of the plasma levels of VEGF-A and PIGF in healthy donors and melanoma patients. Box and whisker plots for the levels of VEGF-A and PIGF in the plasma of healthy donors $(n=8)$ and melanoma patients $(n=8)$, as determined by ELISA. In melanoma patients, blood samples were withdrawn on day 1 of the first cycle of a bevacizumab/paclitaxel/carboplatin regimen, just before drug administration. The top and bottom of each box represent the 75th and 25 th percentile, respectively, and whiskers the 10th and 90th percentiles. The horizontal bar within each box indicates the median. Data were analysed by ANOVA for multiple comparisons followed by Bonferroni test; "P-value $<0.05$. 


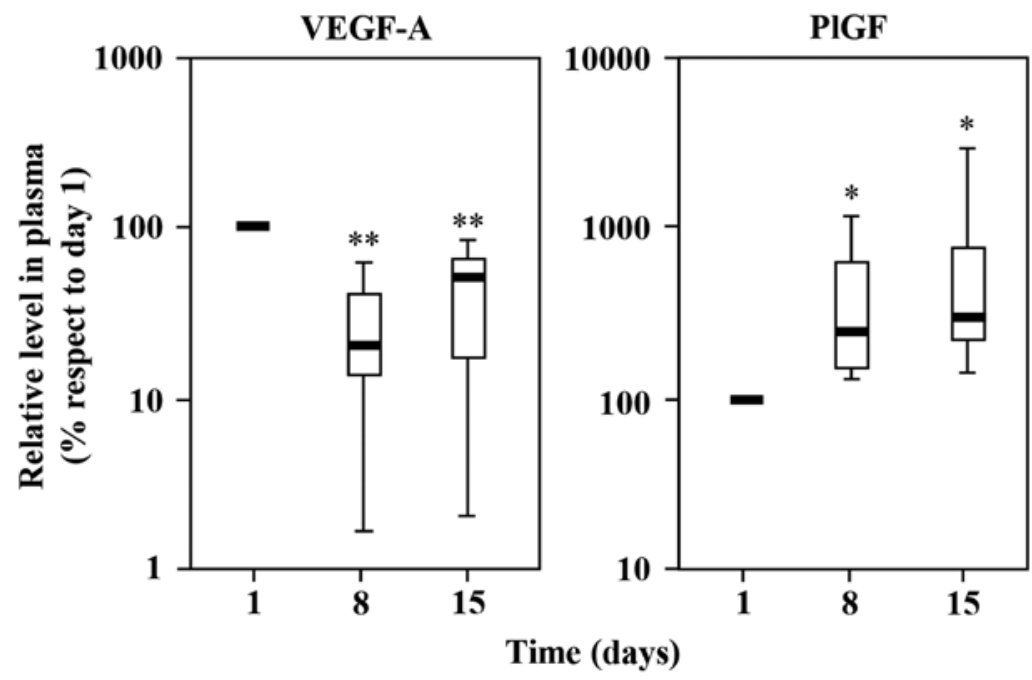

Figure 5. Evaluation of plasma VEGF-A and PlGF levels in melanoma patients during treatment with bevacizumab plus chemotherapy. Box and whisker plots represent the levels of the two growth factors in the plasma on day 8 and on day 15, before drug administration, with respect to the levels found in the plasma of each patient on day 1 before drug administration. Data were analysed by ANOVA for multiple comparisons followed by Bonferroni test; $\mathrm{P}$-values, ${ }^{*} \mathrm{P}<0.05$ and ${ }^{* *} \mathrm{P}<0.01$.

In our melanoma model, we found that cells expressing NRP-1 produce a higher amount of PIGF as compared to the NRP-1 negative cells. PlGF is directly involved in the invasive phenotype of M14-N cells, since neutralizing antibodies reverted ECM invasion in response to the growth factor. Moreover, the chemotactic response of NRP-1 positive cells to exogenous PlGF was abrogated by anti-NRP-1 antibodies, suggesting that a direct interaction of PlGF with NRP-1 is required for PlGF-mediated melanoma invasiveness. Thus, in VEGFR-1 negative cells NRP-1 behaves as a primary receptor capable of transmitting the signal deriving from its binding to PIGF.

Noteworthy, we also found increased levels of PIGF in the plasma of patients with metastatic melanoma as compared to healthy donors. These patients were enrolled in a phase II clinical trial in which bevacizumab was administered in combination with the chemotherapeutic agents paclitaxel and carboplatin. Drug treatment was followed by a transient decrease of VEGF-A and a parallel increase of PIGF plasma levels. In patients with non-small cell lung cancer, treated with a bevacizumab-carboplatin-paclitaxel regimen, a decrease in VEGF-A was also reported and associated with clinical benefit (38). Due to the small number of melanoma patients enrolled in the clinical study, we cannot draw any definitive conclusion about the predictive value of VEGF-A decline and PIGF increase in the plasma of patients. Nevertheless, our results encourage further studies in a larger number of patients to evaluate the role of plasma PIGF as a potential marker of melanoma resistance to anti-VEGF-A treatment.

High levels of PIGF can result in tumour progression through autocrine or paracrine mechanisms. PlGF can be produced not only by tumour cells but also by cells of the tumour stroma (39). Indeed, in a murine in vivo model, the increased plasma levels of PIGF in response to an anti-angiogenic therapy were shown to derive from the host tissues and not from the tumour itself (40). Moreover, using human melanoma cell lines secreting both VEGF and PIGF, we found that treatment with bevacizumab did not cause any modulation of PIGF release in the culture supernatants (data not shown). Therefore, in patients treated with an anti-VEGF-A therapy high plasma levels of PlGF, produced by the tumour or by the stroma, can favour melanoma spreading as long as cancer cells express a significant amount of NRP-1, even in the absence of VEGFR-1.

Considerable experimental evidence allows hypothesizing a potential role for PIGF or NRP-1 as therapeutic targets. Differently from VEGF-A, PIGF plays a marginal role in physiological conditions in adults, but is involved in the induction of the angiogenic switch in pathological conditions, in the mobilization of hematopoietic precursor stem cells from the bone marrow and in the growth and migration of cancer cells (41). Moreover, in preclinical animal models monoclonal antibodies specific for PIGF inhibit the growth and metastasis in different types of tumours and increase the efficacy of chemotherapy without causing significant side effects $(4,6,41)$.

Concerning NRP-1 as therapeutic target, this receptor is expressed in endothelial, highly aggressive melanoma and immune cells and, therefore, is involved in all the biological processes crucial for melanoma progression: angiogenesis, vasculogenic mimicry and tumour evasion from the control of the immune system. Moreover, a high-affinity monoclonal antibody targeting NRP-1 has been shown to inhibit migration of human endothelial cells and tumor formation in animal models $(42,43)$. It also inhibited breast cancer cell proliferation and enhanced chemosensitivity of human non-small cell lung, kidney, prostate cancer and other carcinoma cells, by interfering with integrin-dependent survival pathways (44-46). Thus, the targeting of NRP-1 seems to be a valuable strategy for combination therapies, including anti-angiogenic agents, as reviewed (47).

In conclusion, in this study we have shown that PlGF promotes ECM invasiveness and vasculogenic mimicry of melanoma cells, and that these functions are principally mediated by PIGF activation of NRP-1. Moreover, we found that the plasma levels of PIGF in melanoma patients are higher 
than in healthy donors, and that these levels tend to further increase in the course of a bevacizumab-containing chemotherapy regimen. PlGF production and NRP-1 expression may, therefore, increase tumour aggressiveness and contribute to melanoma escape from anti-VEGF-A therapies. Thus, the blockade of PIGF interaction with its receptors, VEGFR-1 and, more importantly, NRP-1, represents a therapeutic strategy that may enhance the efficacy or counteract resistance to inhibitors of VEGF-A mediated pathways.

\section{Acknowledgements}

This study was supported by the Italian Ministry of Health and in part by the 'Associazione Italiana per la Ricerca sul Cancro' (AIRC) to GG (AIRC 2013 IG 14042).

\section{References}

1. Fischer C, Mazzone M, Jonckx B and Carmeliet P: FLT1 and its ligands VEGFB and PlGF: drug targets for anti-angiogenic therapy? Nat Rev Cancer 8: 942-956, 2008.

2. Roskoski R Jr: VEGF receptor protein-tyrosine kinases: structure and regulation. Biochem Biophys Res Commun 375: 287-291, 2008

3. Lohela M, Bry M, Tammela T and Alitalo K: VEGFs and receptors involved in angiogenesis versus lymphangiogenesis. Curr Opin Cell Biol 21: 154-165, 2009.

4. Dewerchin M and Carmeliet P: Placental growth factor in cancer. Expert Opin Ther Targets 18: 1339-1354, 2014.

5. Kim KJ, Cho CS and Kim WU: Role of placenta growth factor in cancer and inflammation. Exp Mol Med 44: 10-19, 2012.

6. Autiero M, Waltenberger J, Communi D, Kranz A, Moons L, Lambrechts D, Kroll J, Plaisance S, De Mol M, Bono F, et al: Role of PIGF in the intra- and intermolecular cross talk between the VEGF receptors Flt1 and Flk1. Nat Med 9: 936-943, 2003.

7. Marcellini M, De Luca N, Riccioni T, Ciucci A, Orecchia A, Lacal PM, Ruffini F, Pesce M, Cianfarani F, Zambruno G, et al: Increased melanoma growth and metastasis spreading in mice overexpressing placenta growth factor. Am J Pathol 169 643-654, 2006

8. Levati L, Ruffini F, Muzi A, Umezawa K, Graziani G, D'Atri S and Lacal PM: Placenta growth factor induces melanoma resistance to temozolomide through a mechanism that involves the activation of the transcription factor NF- $\mathrm{KB}$. Int J Oncol 38 241-247, 2011.

9. Schwartz JD, Rowinsky EK, Youssoufian H, Pytowski B and $\mathrm{Wu}$ Y: Vascular endothelial growth factor receptor-1 in human cancer: concise review and rationale for development of IMC-18F1 (Human antibody targeting vascular endothelial growth factor receptor-1). Cancer 116 (Suppl): 1027-1032, 2010.

10. Adini A, Kornaga T, Firoozbakht $F$ and Benjamin LE: Placental growth factor is a survival factor for tumor endothelial cells and macrophages. Cancer Res 62: 2749-2752, 2002.

11. Zhou Y, Bellingard V, Feng K-T, McMaster M and Fisher SJ: Human cytotrophoblasts promote endothelial survival and vascular remodeling through secretion of Ang2, PlGF, and VEGF-C. Dev Biol 263: 114-125, 2003.

12. Wu Y, Hooper AT, Zhong Z, Witte L, Bohlen P, Rafii S and Hicklin DJ: The vascular endothelial growth factor receptor (VEGFR-1) supports growth and survival of human breast carcinoma. Int J Cancer 119: 1519-1529, 2006.

13. Wu Y, Zhong Z, Huber J, Bassi R, Finnerty B, Corcoran E, Li H, Navarro E, Balderes P, Jimenez X, et al: Anti-vascular endothelial growth factor receptor-1 antagonist antibody as a therapeutic agent for cancer. Clin Cancer Res 12: 6573-6584, 2006.

14. Fragoso R, Pereira T, Wu Y, Zhu Z, Cabeçadas J and Dias S: VEGFR-1 (FLT-1) activation modulates acute lymphoblastic leukemia localization and survival within the bone marrow, determining the onset of extramedullary disease. Blood 107: 1608-1616, 2006.

15. Lacal PM, Failla CM, Pagani E, Odorisio T, Schietroma C, Falcinelli S, Zambruno G and D'Atri S: Human melanoma cells secrete and respond to placenta growth factor and vascular endothelial growth factor. J Invest Dermatol 115: 1000-1007, 2000.
16. Graells J, Vinyals A, Figueras A, Llorens A, Moreno A, Marcoval J, Gonzalez FJ and Fabra A: Overproduction of VEGF concomitantly expressed with its receptors promotes growth and survival of melanoma cells through MAPK and PI3K signaling. J Invest Dermatol 123: 1151-1161, 2004.

17. Graeven U, Fiedler W, Karpinski S, Ergün S, Kilic N, Rodeck U, Schmiegel W and Hossfeld DK: Melanoma-associated expression of vascular endothelial growth factor and its receptors FLT-1 and KDR. J Cancer Res Clin Oncol 125: 621-629, 1999.

18. Lacal PM, Ruffini F, Pagani E and D'Atri S: An autocrine loop directed by the vascular endothelial growth factor promotes invasiveness of human melanoma cells. Int J Oncol 27: 1625-1632, 2005.

19. Grünewald FS, Prota AE, Giese A and Ballmer-Hofer K: Structure-function analysis of VEGF receptor activation and the role of coreceptors in angiogenic signaling. Biochim Biophys Acta 1804: 567-580, 2010.

20. Neagoe PE, Lemieux C and Sirois MG: Vascular endothelial growth factor (VEGF)-A165-induced prostacyclin synthesis requires the activation of VEGF receptor-1 and -2 heterodimer. J Biol Chem 280: 9904-9912, 2005.

21. Allain B, Jarray R, Borriello L, Leforban B, Dufour S, Liu WQ, Pamonsinlapatham P, Bianco S, Larghero J, Hadj-Slimane R, et al: Neuropilin-1 regulates a new VEGF-induced gene, Phactr-1, which controls tubulogenesis and modulates lamellipodial dynamics in human endothelial cells. Cell Signal 24: 214-223, 2012.

22. Chittenden TW, Claes F, Lanahan AA, Autiero M, Palac RT, Tkachenko EV, Elfenbein A, Ruiz de Almodovar C, Dedkov E, Tomanek R, et al: Selective regulation of arterial branching morphogenesis by synectin. Dev Cell 10: 783-795, 2006.

23. Snuderl M, Batista A, Kirkpatrick ND, Ruiz de Almodovar C, Riedemann L, Walsh EC, Anolik R, Huang Y, Martin JD, Kamoun W, et al: Targeting placental growth factor/neuropilin 1 pathway inhibits growth and spread of medulloblastoma. Cell 152: 1065-1076, 2013.

24. Ellis LM: The role of neuropilins in cancer. Mol Cancer Ther 5: 1099-1107, 2006.

25. Holmes K, Roberts OL, Thomas AM and Cross MJ: Vascular endothelial growth factor receptor-2: structure, function, intracellular signalling and therapeutic inhibition. Cell Signal 19: 2003-2012, 2007

26. van Beijnum JR, Nowak-Sliwinska P, Huijbers EJ, Thijssen VL and Griffioen AW: The great escape; the hallmarks of resistance to antiangiogenic therapy. Pharmacol Rev 67: 441-461, 2015.

27. Lambrechts D, Lenz HJ, de Haas S, Carmeliet P and Scherer SJ: Markers of response for the antiangiogenic agent bevacizumab. J Clin Oncol 31: 1219-1230, 2013.

28. Ruffini F, D'Atri S and Lacal PM: Neuropilin-1 expression promotes invasiveness of melanoma cells through vascular endothelial growth factor receptor-2-dependent and -independent mechanisms. Int J Oncol 43: 297-306, 2013.

29. Ruffini F, Graziani G, Levati L, Tentori L, D'Atri S and Lacal PM: Cilengitide downmodulates invasiveness and vasculogenic mimicry of neuropilin 1 expressing melanoma cells through the inhibition of $\alpha v \beta 5$ integrin. Int J Cancer 136: E545-E558, 2015.

30. Orecchia A, Lacal PM, Schietroma C, Morea V, Zambruno G and Failla CM: Vascular endothelial growth factor receptor-1 is deposited in the extracellular matrix by endothelial cells and is a ligand for the alpha 5 beta 1 integrin. J Cell Sci 116: 3479-3489, 2003.

31. Ruffini F, Tentori L, Dorio AS, Arcelli D, D'Amati G, D'Atri S, Graziani $\mathrm{G}$ and Lacal PM: Platelet-derived growth factor $\mathrm{C}$ and calpain-3 are modulators of human melanoma cell invasiveness. Oncol Rep 30: 2887-2896, 2013.

32. Seftor RE, Hess AR, Seftor EA, Kirschmann DA, Hardy KM, Margaryan NV and Hendrix MJ: Tumor cell vasculogenic mimicry: From controversy to therapeutic promise. Am J Pathol 181: 1115-1125, 2012.

33. Escudero-Esparza A, Martin TA, Douglas-Jones A, Mansel RE and Jiang WG: PGF isoforms, PLGF-1 and PGF-2 and the PGF receptor, neuropilin, in human breast cancer: prognostic significance. Oncol Rep 23: 537-544, 2010.

34. Banerjee S, Sengupta K, Dhar K, Mehta S, D'Amore PA, Dhar G and Banerjee SK: Breast cancer cells secreted platelet-derived growth factor-induced motility of vascular smooth muscle cells is mediated through neuropilin-1. Mol Carcinog 45: 871-880, 2006.

35. Dhar K, Dhar G, Majumder M, Haque I, Mehta S, Van Veldhuizen PJ, Banerjee SK and Banerjee S: Tumor cell-derived PDGF-B potentiates mouse mesenchymal stem cells-pericytes transition and recruitment through an interaction with NRP-1. Mol Cancer 9: 209, 2010. 
36. Pellet-Many C, Frankel P, Evans IM, Herzog B, JünemannRamírez M and Zachary IC: Neuropilin-1 mediates PDGF stimulation of vascular smooth muscle cell migration and signalling via p130Cas. Biochem J 435: 609-618, 2011.

37. Frank A, David V, Aurelie TR, Florent G, William H and Philippe B: Regulation of MMPs during melanoma progression: from genetic to epigenetic. Anticancer Agents Med Chem 12: 773-782, 2012.

38. Tamiya M, Tamiya A, Yamadori T, Nakao K, Asami K, Yasue T, Otsuka T, Shiroyama T, Morishita N, Suzuki H, et al: Phase2 study of bevacizumab with carboplatin-paclitaxel for non-small cell lung cancer with malignant pleural effusion. Med Oncol 30: 676, 2013.

39. Jayasinghe C, Simiantonaki N and Kirkpatrick CJ: Cell typeand tumor zone-specific expression of pVEGFR-1 and its ligands influence colon cancer metastasis. BMC Cancer 15: $104,2015$.

40. Bagley RG, Ren Y, Weber W, Yao M, Kurtzberg L, Pinckney J, Bangari D, Nguyen C, Brondyk W, Kaplan J, et al: Placental growth factor upregulation is a host response to antiangiogenic therapy. Clin Cancer Res 17: 976-988, 2011.

41. Fischer C, Jonckx B, Mazzone M, Zacchigna S, Loges S, Pattarini L, Chorianopoulos E, Liesenborghs L, Koch M, De Mol M, et al: Anti-PlGF inhibits growth of VEGF(R)-inhibitor-resistant tumors without affecting healthy vessels. Cell 131: 463-475, 2007.
42. Liang WC, Dennis MS, Stawicki S, Chanthery Y, Pan Q, Chen Y, Eigenbrot C, Yin J, Koch AW, Wu X, et al: Function blocking antibodies to neuropilin-1 generated from a designed human synthetic antibody phage library. J Mol Biol 366: 815-829, 2007.

43. Pan Q, Chanthery Y, Liang WC, Stawicki S, Mak J, Rathore N, Tong RK, Kowalski J, Yee SF, Pacheco G, et al: Blocking neuropilin-1 function has an additive effect with anti-VEGF to inhibit tumor growth. Cancer Cell 11: 53-67, 2007.

44. Xin Y, Li J, Wu J, Kinard R, Weekes CD, Patnaik A, Lorusso P, Brachmann R, Tong RK, Yan Y, et al: Pharmacokinetic and pharmacodynamic analysis of circulating biomarkers of anti-NRP1, a novel antiangiogenesis agent, in two phase I trials in patients with advanced solid tumors. Clin Cancer Res 18: 6040-6048, 2012.

45. Jia H, Cheng L, Tickner M, Bagherzadeh A, Selwood D and Zachary I: Neuropilin-1 antagonism in human carcinoma cells inhibits migration and enhances chemosensitivity. Br J Cancer 102: 541-552, 2010.

46. Zeng F, Luo F, Lv S, Zhang H, Cao C, Chen X, Wang S, Li Z, Wang X, Dou X, et al: A monoclonal antibody targeting neuropilin-1 inhibits adhesion of MCF7 breast cancer cells to fibronectin by suppressing the FAK/p130cas signaling pathway. Anticancer Drugs 25: 663-672, 2014.

47. Graziani G and Lacal PM: Neuropilin-1 as therapeutic target for malignant melanoma. Front Oncol 5: 125, 2015. 